\title{
ATP-dependent nucleosome disruption at a heat-shock promoter mediated by binding of GAGA transcription factor
}

\author{
Toshio Tsukiyama, Peter B. Becker ${ }^{*}$ \& Carl $\mathrm{Wu}^{\dagger}$ \\ Laboratory of Biochemistry, National Cancer Institute, National Institutes of Health, Building 37, Room 4C-09, Bethesda, Maryland 20892, USA
}

Genetic control elements are usually situated in local regions of chromatin that are hypersensitive to structural probes such as DNase I. We have reconstructed the chromatin structure of the hsp70 promoter using an in vitro nucleosome assembly system. Binding of the GAGA transcription factor on existing nucleosomes leads to nucleosome disruption, DNase I hypersensitivity at the TATA box and heat-shock elements, and rearrangement of adjacent nucleosomes. ATP hydrolysis facilitates this process, suggesting that an energy-dependent pathway is involved in chromatin remodelling.

THE role of nucleosomes as general repressors of transcriptional initiation in eukaryotic cells is well established ${ }^{1-3}$, but the mechanisms by which transcription factors, enhancer proteins and RNA polymerases gain access to target sequences in chromatin remain poorly understood. In vivo, genetic control elements are usually situated in accessible, nuclease-hypersensitive sites that punctuate the orderly array of nucleosomes on the chromatin fibre $^{4,5}$. Generation of these accessible regions in chromatin seems to be a prerequisite for the formation of an active transcription complex, and may involve a class of transcription factors whose binding alters the stability of underlying or adjacent nucleosomes ${ }^{6.7}$

We have developed an in vitro assay for the establishment of a nucleosome-free region in chromatin, using a chromatin assembly extract prepared from Drosophila embryos $^{8}$, plasmid DNA, and purified transcription factors. As a model system, we analysed the promoter of the Drosophila hsp 70 gene encoding heat-shock protein 70 . The promoter contains sites for interaction with HSF, the heat-shock transcription factor ${ }^{9}$, and GAGA, a constitutively expressed transcription factor that binds to GA/CT-rich sites present in many Drosophila genes ${ }^{10,11}$, and for TFIID, the TATA-binding general transcription factor complex $^{12}$; it is also bound to an RNA polymerase II molecule that has paused after synthesizing a short transcript ${ }^{13.14}$. GAGA factor, TFIID and RNA polymerase II are active and can associate with heat-shock promoters in vitro in the absence of a heatshock stimulus ${ }^{15}{ }^{18}$, so they are potential candidates for establishing an accessible promoter complex poised to respond to the binding of the activated, trimeric form of $\mathrm{HSF}^{9}$. Here we describe the role of the GAGA factor in altering chromatin structure. We show that the introduction of GAGA protein during or after nucleosome assembly in vitro results in a disruption of nucleosome structure at the hsp 70 promoter. The disruption is characterized by hypersensitivity to DNase I digestion and a realignment of adjacent nucleosomes, and is facilitated by the presence of hydrolysable ATP.

\section{Disruption of chromatin by GAGA factor}

We analysed the ability of GAGA transcription factor ${ }^{10}$ to disrupt nucleosome organization using affinity-purified, histidinetagged GAGA protein expressed in insect cells with a baculovirus expression vector. Recombinant GAGA factor (hereafter called GAGA) was introduced at each of three stages of nucleosome assembly on a 6.2-kilobase (kb) plasmid carrying $h s p 70$ :

* Present address: Gene Expression Programme, EMBL, Meyerhofstrasse 1, D-69117 Heidel-

berg. Germany.
t To whom correspondence should be addressed. at the onset (zero time, $0 \mathrm{~h}$ ), at a stage when the assembly of regularly spaced nucleosomes is nearly complete $(2.5 \mathrm{~h})$, or when nucleosome assembly has reached a maximum $(5.5 \mathrm{~h})$ (ref. 8 , and our unpublished observations). The reconstituted plasmid chromatin was tested for the presence of nucleosome organization at specific locations by prolonged digestion with micrococcal nuclease (MNase), followed by gel electrophoresis and blot hybridization with unique oligonucleotide probes. As micrococcal nuclease initially cleaves within the linker DNA between nucleosome core particles and then progressively trims to the core from each end of the nucleosome ${ }^{19}$, the presence of a nucleosome core particle can be gauged by the accumulation of the canonical, 146-base-pair (bp) nuclease-resistant fragment surviving near the limit of digestion.

When the $h s p 70$-plasmid DNA was reconstituted in the chromatin assembly reaction without GAGA, the micrococcal nuclease digestion pattern of the promoter region revealed by hybridization (Fig. 1a) clearly shows that an intact nucleosome has been assembled at sequences corresponding to the oligonucleotide probe (the probe is specific for DNA between positions 115 to 132 , which partially overlaps two of four GA/CT elements on the $h s p 70$ promoter) (see also Fig. $1 d$, probe C). In addition to the 146-bp fragment derived from the nucleosome core particle, a ladder of discrete fragments corresponding to nucleosome oligomers can be seen at intermediate stages of digestion by micrococcal nuclease. This pattern of cleavage indicates that the DNA surrounding the $h s p 70$ promoter is organized in a regularly spaced (but not necessarily positioned) array of nucleosomes with a characteristic repeat length of $\sim 180 \mathrm{bp}^{8}$.

A dramatically different cleavage pattern at the $h s p 70$ promoter was seen when GAGA was added at the onset of nucleosome assembly $(0 \mathrm{~h})$ in (Fig. $1 a$ ). Upon extensive digestion with micrococcal nuclease, the abundance of the 146-bp fragment is decreased up to 5-fold. In addition, subnucleosomal fragments shorter than $146 \mathrm{bp}$ are evident, despite the difficulty in effecting quantitative Southern transfer of very small DNAs. The generation of these subfragments and the loss of the 146-bp fragment represents an invasion and cleavage of the DNA within the nucleosome core particle by micrococcal nuclease, showing that nucleosome organization has been disrupted at the promoter region and giving rise to a smear of DNA sizes at intermediate stages of digestion (Fig. 1a), rather than the repeat pattern of nucleosome oligomers. This ability of the nuclease to invade the disrupted region is probably a result of the secondary trimming action of the enzyme rather than of primary endonucleolytic cleavage (see later).

Using these criteria for nucleosome disruption, chromatin 
FIG. 1 GAGA-dependent chromatin disruption in vitro. a-c, Micrococca nuclease (MNase) digestion patterns of hsp70-plasmid chromatin reconstituted with GAGA as indicated. DNA blots were hybridized sequentially with oligonucleotides: $a,(-115$ to -132$) ; b,(+1,803$ to $+1,832)$; and $c,\left(2,499\right.$ to 2,528 of pBluescript SK $\left.{ }^{-}\right)$, respectively. Numbers to the left indicate size calibration (in base pairs). $d$, MNase digestion patterns of the $h s p 70$ promoter region reconstituted with GAGA
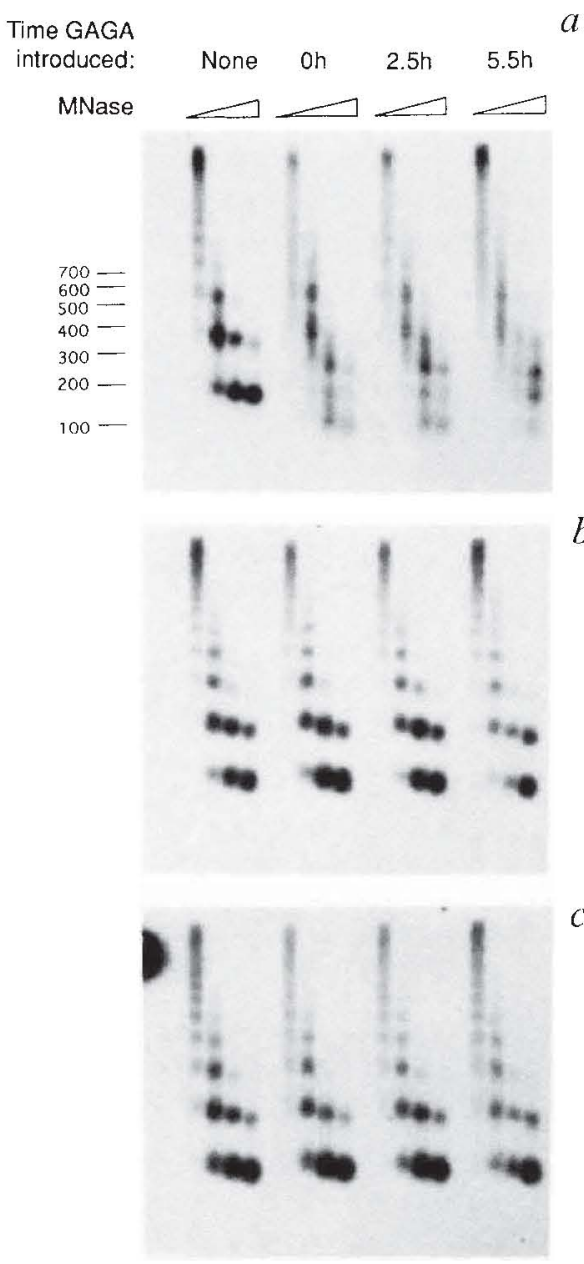

added at $2.5 \mathrm{~h}$ of nucleosome assembly. The blot was sequentially hybridized with the probes A-G (map positions: $A,-340$ to -311 ; $B$, -184 to $-165,-132$ to $-115, \mathrm{D},-89$ to $-50, \mathrm{E},-36$ to -17 ; F +19 to $+36 ; G,+148$ to +176 ). Similar results were obtained when GAGA was added at $\mathrm{O} \mathrm{h}$ or after $5.5 \mathrm{~h}$ of assembly. $e$, Antibody inhibition of GAGA function.

METHODS. Chromatin assembly extracts were prepared from preblastoderm stage embryos as described ${ }^{8}$. The hsp70-plasmid (pdhspXX3.2) was constructed from a 3.2-kb Xbal fragment of $\mathrm{p} 122 \times 14$ (ref. 49) inserted in pBluescript $\mathrm{SK}^{-}$. In a typical reaction, $100 \mathrm{ng}$ plasmid DNA and $650 \mathrm{ng}$ ФX174 DNA were incubated in $100 \mu \mathrm{l}$ for $6 \mathrm{~h}$ at $26^{\circ} \mathrm{C}$ as described ${ }^{\circ}$. GAGA protein was expressed using the baculovirus vector pBlueBacHisB (Invitrogen). The 1.6-kb EcoRI fragment carrying the GAGA cDNA was cleaved from pAc-GAGA ${ }^{10}$ and inserted in the multiple cloning site of pBluescript $\mathrm{SK}^{-}$. The cDNA fragment was then released by cleavage with BamHI and Hindlll and inserted in pBlueBacHisB. The recombinant GAGA protein lacks five natural residues at the $\mathrm{N}$ terminus, and gains 37 residues, including six histidines from the vector ${ }^{10}$. After expression for 2 days in Sf9 cells, GAGA was extracted from cell lysates in buffer containing $20 \mathrm{mM}$ Tris- $\mathrm{HCl}$, pH 8.0, $5 \mathrm{mM} \mathrm{MgCl}_{2}, 0.4 \mathrm{M} \mathrm{NaCl}$, $5 \%$ glycerol, $1 \mathrm{mM}$ 3-mercaptoethanol, $0.2 \mathrm{mM}$ PMSF, and purified through DEAE-Sepharose CL6B (Pharmacia). The flow-through and wash fractions were applied to $\mathrm{Ni}^{2+}$-NTA-agarose (Qiagen), and GAGA was eluted with buffer containing $100 \mathrm{mM}$ imidazole, followed by concentration with a Centricon 100 (Amicon) filter. The purity of the GAGA prepa- $e$

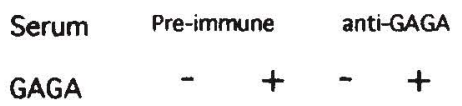

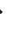

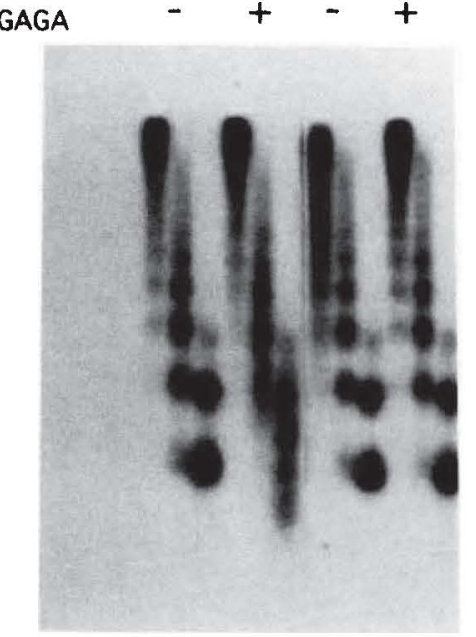
ration was determined by SDS-PAGE and Coomassie blue staining to be $\sim 10 \%$. The amount of GAGA able to disrupt chromatin at $5.5 \mathrm{~h}$ of assembly was determined by titration to be about equivalent to footprinting quantities. Assembled templates were digested with MNase as before ${ }^{8}$ and DNA was processed for Southern blotting and hybridized as described (P.B.B., T.T. and C.W., manuscript in preparation). Polyclonal antibodies against GAGA were prepared in rabbits from protein expressed in $E$. coli ${ }^{10}$ and purified to $>95 \%$ homogeneity on heparin-Sepharose CL6B and by preparative SDSPAGE. Preimmune and immune sera ( $5 \mu \mathrm{l}$ each) were added with GAGA to a $50-\mu$ l assembly reaction at $\mathrm{Oh}$.

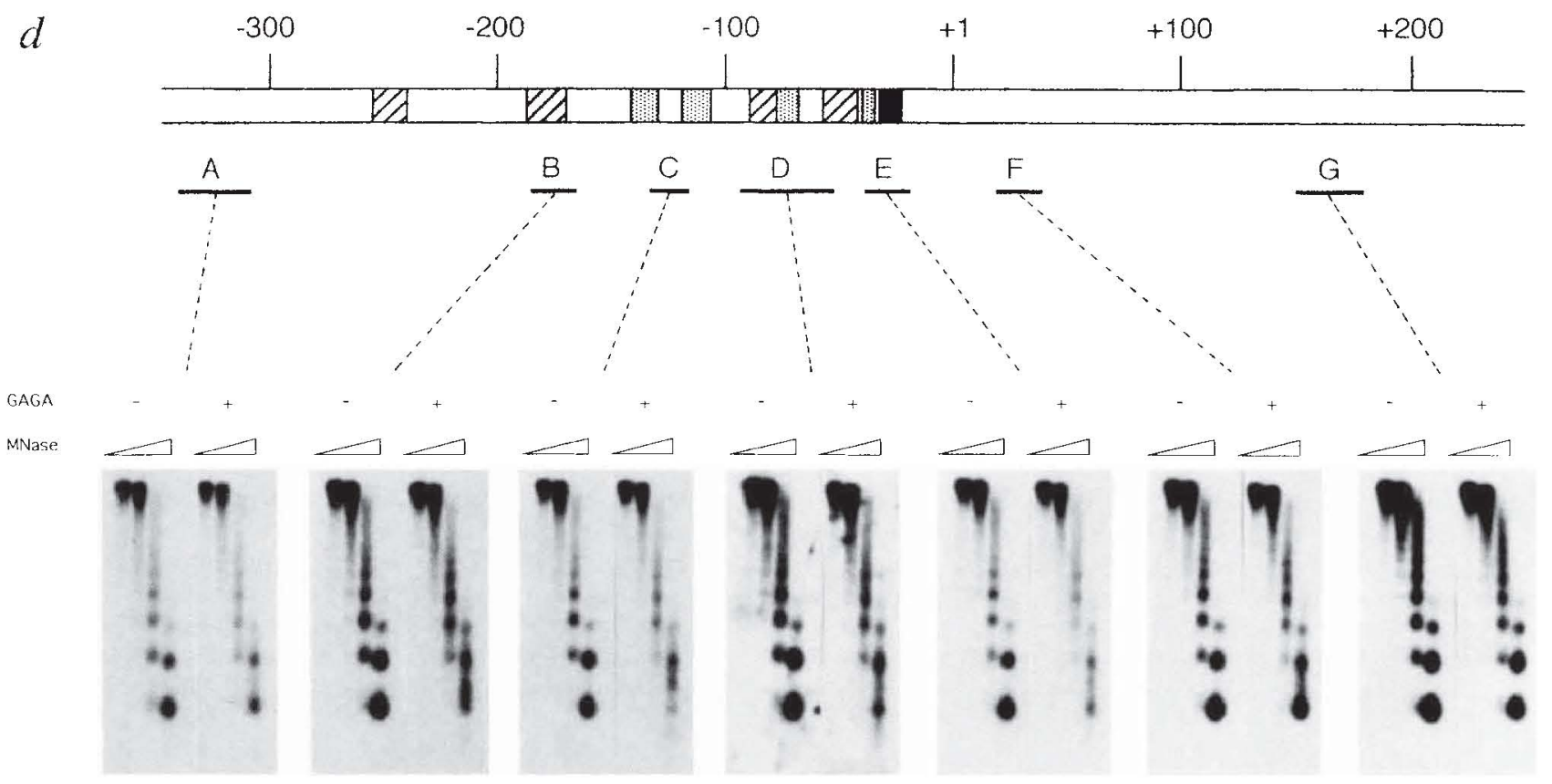


structure was similarly altered when GAGA was introduced after $2.5 \mathrm{~h}$ of nucleosome assembly, and was also significantly affected when GAGA was introduced after the completion of nucleosome assembly (at $5.5 \mathrm{~h}$; Fig. 1 a). We conclude that GAGA can alter the structure of nucleosomes whether by direct competition with the process of nucleosome assembly in vitro, or by affecting the structure of nucleosomes previously deposited on DNA. To investigate the effects of GAGA on chromatin structure at locations distant from the $h s p 70$ promoter, the same DNA blot was stripped of the probe and rehybridized with oligonucleotides
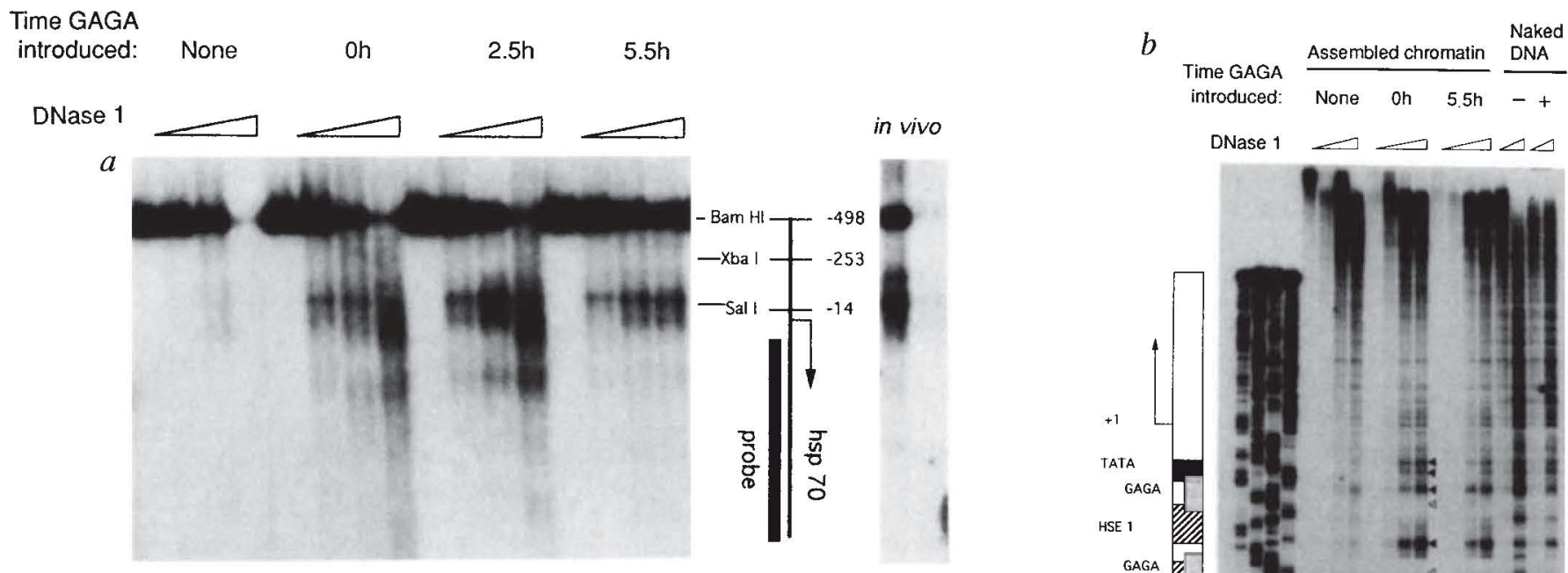

$c$

Time GAGA

introduced: None on $2.5 \mathrm{~h} \quad 5.5 \mathrm{~h}$

MNase

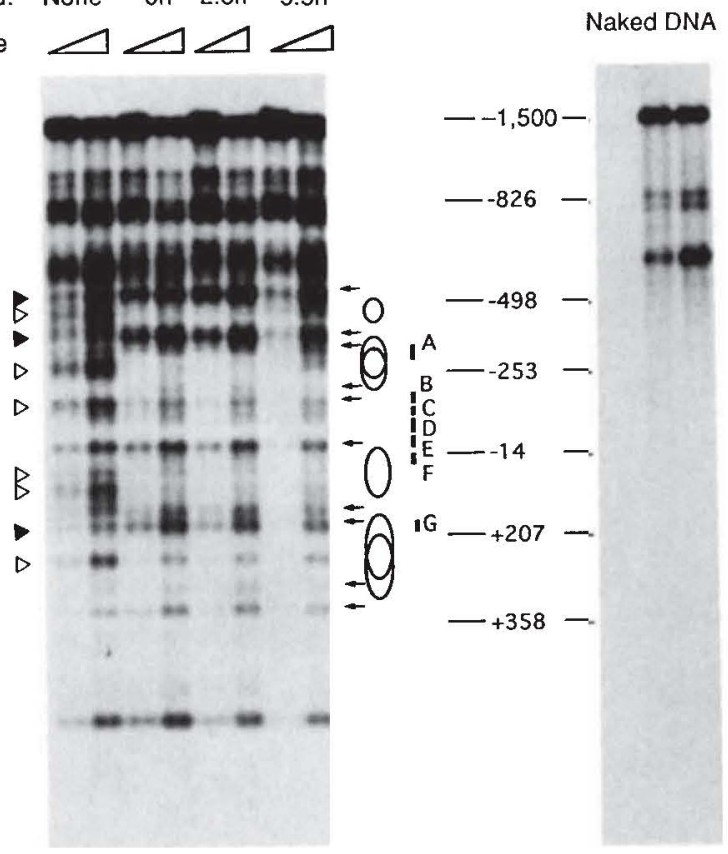

FIG. 2 Mapping DNase I hypersensitivity and nucleosome positions on the reconstituted $h s p 70$ promoter. $a$, Indirect end-labelling of DNase I cleavages relative to a BamHI site at $+1,258$ (ref. 20). The location of the probe on a restriction map of $h s p 70$ is indicated by the solid bar. The in vivo sample shows the cleavage pattern of the endogenous hsp70 genes in $0-24 \mathrm{~h}$ embryo nuclei. $b$, Primer extension-linear amplification analysis of DNase / cleavages on a $6 \%$ sequencing gel. Hypersensitive nucleotides are indicated by filled triangles, and protected nucleotides by open triangles. The GAGA binding sites, heatshock elements (HSE) and TATA box are indicated by the stippled, hatched, and solid bars. The first four lanes on the left are sequencing reactions: T, C, G, A. C, Indirect end-labelling of MNase cleavages relative to a Pstl restriction site at $+1,187$. Arrows connect MNase cleavages adjacent to the promoter region (minor cleavages are not included) with the assigned locations of core particles. Open triangles, suppressed cleavages; filled triangles, enhanced cleavages. The predominant nucleosome positions (including overlaps) are indicated by the open circles; the positions of oligonucleotide probes used in Fig. $1 d$ are indicated by solid bars.

METHODS. hsp70-plasmid chromatin was reconstituted as for Fig. 1c$e$ and digested with $0.1-1.0$ units $\mu l^{-1}$ of DNase I for $1 \mathrm{~min}$ at room temperature. Purified DNA was digested with BamHI, which cuts at $+1,258$ and at -498 , separated on a $1.2 \%$ agarose gel, and transferred to a GeneScreen membrane. The blot was probed with a ${ }^{32} \mathrm{P}$-labelled Nrul $(+32)$ to Pstl $(+1,187)$ fragment. For linear amplification, a ${ }^{32} \mathrm{P}$ labelled primer $(-184$ to -165$)$ was extended with Taq polymerase for 30 cycles using the Cycle Sequencing Kit (Perkin Elmer). The reaction was supplemented with dNTPs ( $10 \mu \mathrm{M}$ each) in $5 \mu$ l (final) according to the manufacturer's directions. For naked DNA controls, the same amount of GAGA was incubated with plasmid DNA before nuclease digestion. For mapping MNase cleavages by indirect end-labelling, DNA from the MNase digestion samples of Fig. 1d were cleaved with Pstl $(+1,187)$ and Sacll (which cuts in the multiple cloning site at nucleotide 751 of pBluescript, just upstream of the Xbal site at $-1,515$ ). DNA was separated on a $1.1 \%$ agarose gel $(24 \mathrm{~h}$ electrophoresis in TBE buffer at $1.5 \mathrm{~V}$ per $\mathrm{cm}$ ) and the blot was hybridized with an Nrul-Pstl fragment. 
corresponding to the $3^{\prime}$ end of the $h s p 70$ gene and to the ampicillin-resistance gene of the plasmid vector (Fig. $1 b$ and $c$ ). GAGA had no effect on micrococcal nuclease digestion patterns in either case, demonstrating the specificity of GAGA-mediated nucleosome disruption; these controls were used as an internal standard for quantifying changes in the micrococcal nuclease digestion pattern at the $h s p 70$ promoter region.

We next determined the extent of specific disruption along hsp70 promoter sequences by sequential blot hybridization with oligonucleotide probes spanning the entire promoter from positions -340 to +176 (Fig. $1 d$; probes $\mathrm{A}-\mathrm{G}$ ). Among these, probes B-E, which cover the upstream region from -184 to -17 (including the four $\mathrm{GA} / \mathrm{CT}$ elements), revealed significant nucleosome disruption as evidenced by GAGA-dependent loss of the nucleosome monomer fragment upon extensive digestion, with generation of subfragments and a smearing of the nucleosome oligomer ladder. The extent of DNA spanned by these probes $(\sim 160 \mathrm{bp})$ indicates that histone DNA interactions in the nucleosome core are disturbed over this region, presumably as a result of GAGA binding. We observed a smaller effect of GAGA on the digestion pattern for probes $A, F$ and $G$; the nucleosomes on these regions are rearranged at a number of restricted locations (see below). To confirm that the disruption of nucleosomes on the $h s p 70$ promoter was dependent on GAGA and not on other proteins present in the GAGA preparation, we showed that disruption was abolished by the presence of polyclonal antibodies raised against purified bacterially expressed GAGA (Fig. 1e).

\section{Reconstitution of DNase I hypersensitivity}

As revealed by partial DNase I digestion and indirect end-labelling, a broad hypersensitive site with a major peak at about position -100 is reconstituted on the $h s p 70$ promoter when GAGA is introduced in the assembly reaction (Fig. 2a). DNase I hypersensitivity is observed when GAGA was added at the start $(0 \mathrm{~h})$, at $2.5 \mathrm{~h}$, and at the completion of nucleosome assembly $(5.5 \mathrm{~h})$. Although the major hypersensitive peak at about position -100 is close to or coincident with the natural peak of hypersensitivity at around position -93 previously mapped on the endogenous $h s p 70$ genes ${ }^{20}$ (Fig. $2 a$ : 'in vivo'), some differences in the fine structure and span of the hypersensitive region can be seen. As the basal transcription factors and RNA polymerase II are deficient in the chromatin assembly extract (unpublished observations), the inclusion of these components may be necessary to reconstitute the whole hypersensitive structure faithfully.

The positions of DNase I cleavage were mapped to singlenucleotide resolution by primer extension-linear amplification analysis (Fig. $2 b$ ). Protection from DNase I cleavage was moderate over the GA/CT repeats when GAGA was introduced in the nucleosome assembly reaction, but the sequences between and flanking the GA/CT repeats were strongly hypersensitive (Fig. $2 b$ ). The TATA box and two heat-shock control elements are included in the sequences hypersensitive to DNase I. Positions of hypersensitive cleavage are consistent with the low-resolution map of the DNase I-hypersensitive site obtained by indirect end-labelling, and with the region of nucleosome disrup-

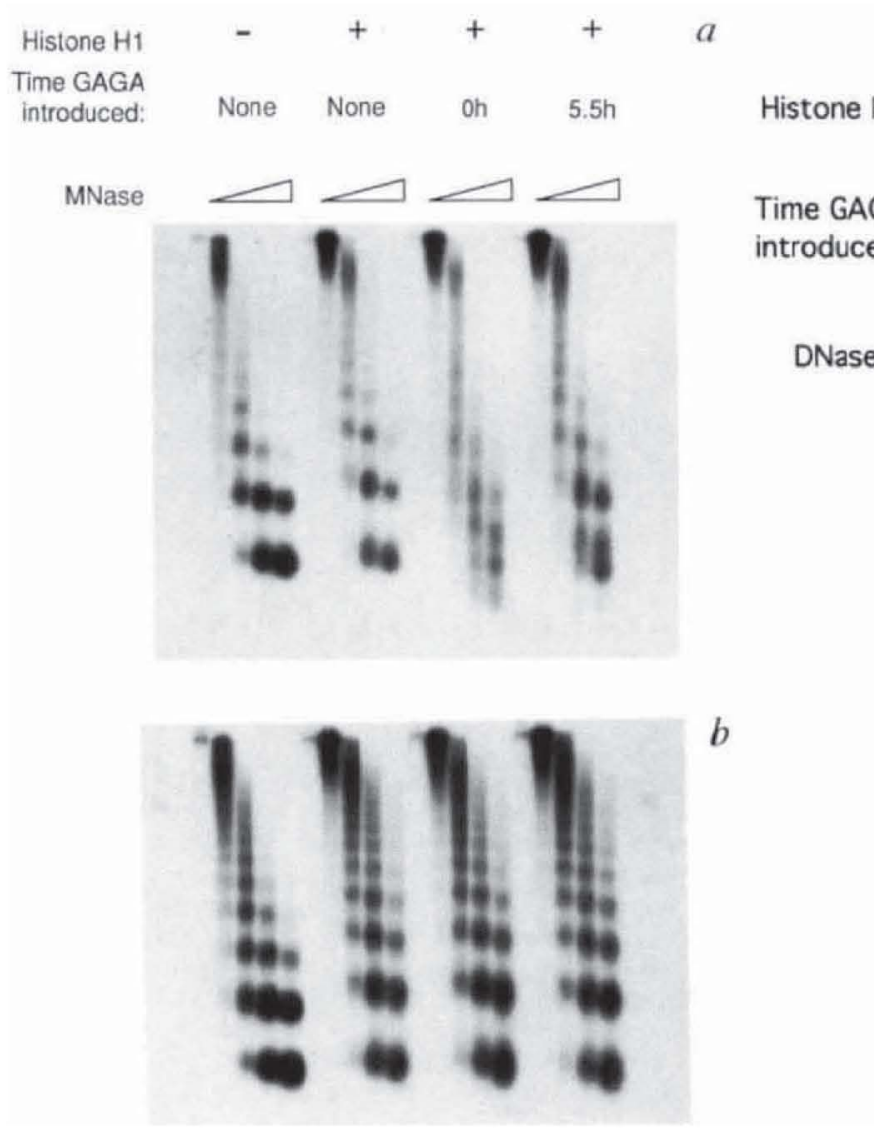

FIG. 3 Effect of GAGA on histone H1-containing chromatin. $a, b$, MNase digestion patterns of hsp 70 plasmid chromatin reconstituted with GAGA and histone $\mathrm{H} 1$ as indicated. DNA was blotted and hybridized sequentially with oligonucleotides: $a,(-115$ to -132$)$, and $b,(+1,803$ to
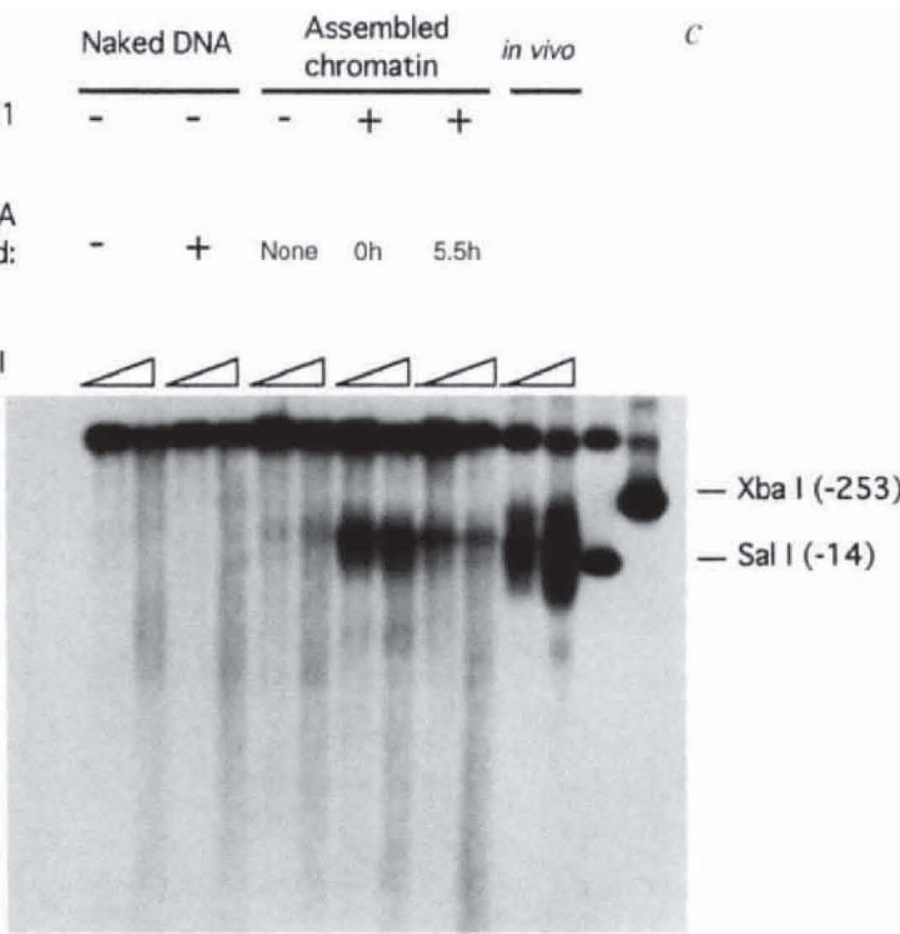

$+1,832$ ). $c$, Indirect end-labelling of DNase I cleavages of naked DNA or hsp 70 chromatin assembled with histone $\mathrm{H} 1$ and GAGA, as indicated. Locations of upsteam $X$ bal and Sall sites are shown.

METHODS. Histone $\mathrm{H} 1$ was purified from $0-12$ h Drosophila embryos almost to homogeneity as described ${ }^{50}$. The amount of $\mathrm{H} 1$ protein added to the assembly reaction was titrated so that the average nucleosome repeat was increased to $\sim 195 \mathrm{bp}$; the amount of $\mathrm{H} 1$ incorporated, as estimated by silver staining, is roughly stoichiometric ${ }^{8}$. The reconstitution assay and subsequent analysis are described in the legends to Figs 1 and 2 . 
tion determined by extensive digestion with micrococcal nuclease.

\section{Rearrangement of adjacent nucleosomes}

We determined the positions of nucleosomes assembled in the vicinity of the $h s p 70$ promoter by mapping the nucleosome linker regions after partial digestion with micrococcoal nuclease digestion and indirect end-labelling (Fig. $2 c$ ). When the $h s p 70$-carrying plasmid was reconstituted in the absence of GAGA, the initial nuclease cleavages in the vicinity of the $h s p 70$ promoter were spaced irregularly, reflecting substantial heterogeneity in the positions of the assembled nucleosomes. Inclusion of GAGA at the start, at $2.5 \mathrm{~h}$, or at the completion of nucleosome assembly resulted in suppression and enhancement of cleavages in the regions flanking the site of nucleosome disruption. Changes in the initial cleavage pattern after GAGA binding allow new assignments for the predominant nucleosome positions surrounding the $h s p 70$ promoter. Hence, as well as disrupting nucleosome organization over its cognate sites, binding of GAGA causes realignment of surrounding nucleosomes, probably by restricting adjacent nucleosomes to a subset of positions. The micrococcal nuclease digestion patterns for oligonucleotide probes $\mathrm{A}, \mathrm{F}$ and $\mathrm{G}$ (Fig. 1d) are consistent with their locations in relation to the rearranged nucleosome core and linker positions. As already mentioned, initial cleavages by micrococcal nuclease mapped by indirect end-labelling do not reveal nucleosome disruption at the promoter as hypersensitivity to the nuclease; in fact, the initial cleavage pattern demonstrates some protection of the promoter region upon GAGA binding.

\section{Effect on histone H1-containing chromatin}

Nucleosomes assembled with the preblastoderm Drosophila embryo extract are deficient in the major linker histone $\mathrm{H} 1$, which is apparently synthesized only during post blastoderm development ${ }^{21}$. We have shown previously that purified histone H1 can be incorporated during nucleosome assembly, leading to an increased repeat length of $\sim 197 \mathrm{bp}^{8}$. Under these assembly conditions, GAGA is effective in specifically disrupting nucleosome structure on the $h s p 70$ promoter when introduced at the onset of assembly (Fig. 3a, b). But when GAGA is added at the completion of assembly ( $5.5 \mathrm{~h}$ ), disruption is less (Fig. $3 a$ ). Disruption of $\mathrm{Hl}$-containing chromatin was accompanied by the formation of a DNAse I-hypersensitive site, which was less prominent when GAGA was introduced at the completion of assembly (Fig. 3c). Thus the ability of GAGA to dislodge preassembled nucleosomes at the hsp 70 promoter is attenuated in chromatin containing histone $\mathrm{H} 1$.

\section{Promoter requirements for disruption}

We tested the sequence requirements for nucleosome disruption by reconstituting plasmids carrying deletions of the hsp70 upstream region (Fig. 4a). The effect of GAGA on plasmid pdhsp $\Delta 186$, which includes the four GA/CT elements within sequences -186 to +296 of the $h s p 70$ gene, was essentially the same as the effect on the original $h s p 70$ plasmid containing the entire coding region and $1.5 \mathrm{~kb}$ of upstream DNA (Fig. $4 b$ ). But when the template carried a $5^{\prime}$ deletion to -90 , removing the two distal GA/CT elements (pdhsp $\Delta 90$ ), there was disruption when GAGA was added at the start but not at the completion $a$

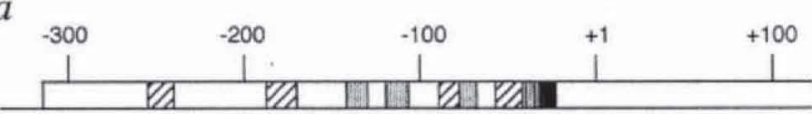

$\triangle 186$

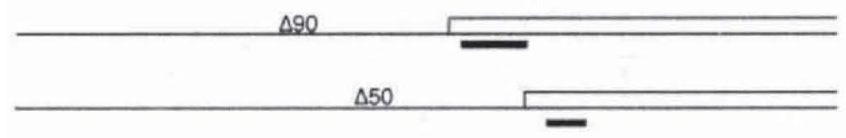

TATA box

ED HSE

GAGA sites

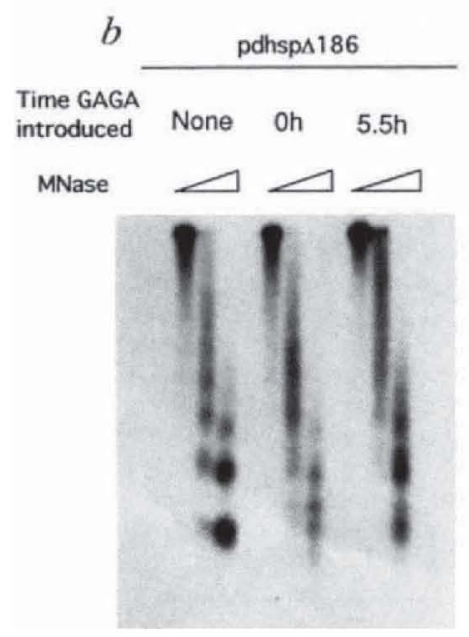

c

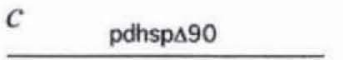

$d$ disruption

o h $\quad 5.5 \mathrm{~h}$
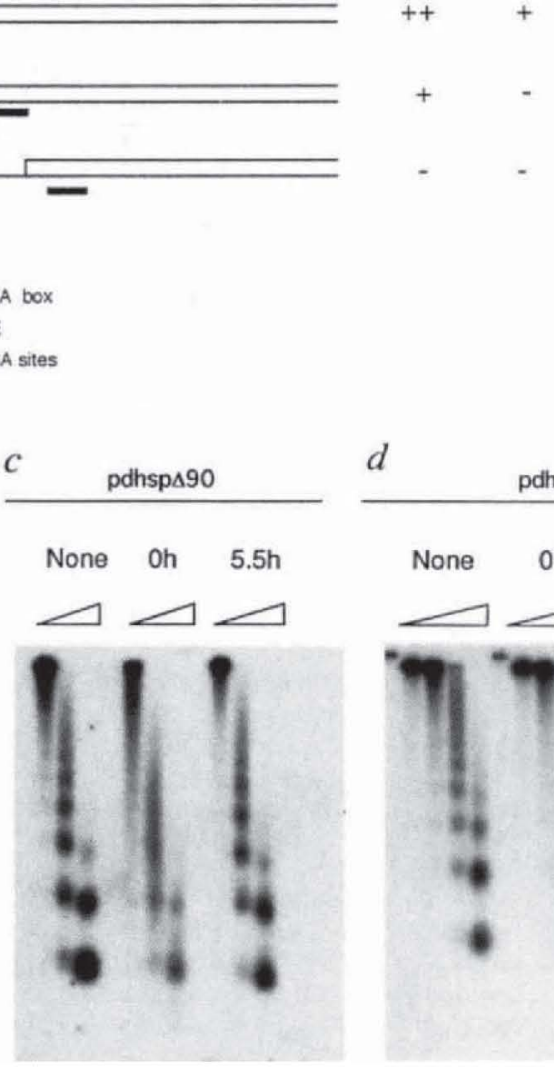

FIG. 4 Promoter elements for chromatin disruption. a, Restriction map showing hsp 70 promoter deletions. Vector DNA is represented by the thin line, and open bars show the hsp70 gene. Oligonucleotide probes used for each plasmid template are indicated by solid bars. All constructs contain up to $+296 \mathrm{bp}$ of the hsp70 sequence. The relative extent of chromatin disruption is summarized on the right; $(++)$ is the designated level of disruption observed on plasmid dhspXX3.2, which carries $1.5 \mathrm{~kb}$ of upstream DNA. $b-d$, Effects of GAGA on the assembly of $h s p 70$ promoter constructs carrying upstream sequences to -186 (pdhsp $\Delta 186), \quad-90 \quad$ (pdhsp $\Delta 90)$ and -50 (pdhsp 450 ), respectively. Plasmid DNAs were reconstituted with GAGA, digested with MNase, and DNA blots were hybridized with oligonucleotides $\mathrm{C}$ $\mathrm{D}$ and $\mathrm{E}$ as for Fig. 1. METHODS. Assembly reactions and subsequent analyses are described in Fig. 1 legend, except that $50 \mathrm{ng}$ promoter plasmid DNA and $700 \mathrm{ng}$ ФX174 DNA were used. 
of assembly (Fig. $4 c$ ). When the $h s p 70$ promoter was deleted to -50 , which removes the third GA/CT element ( $\operatorname{pdhsp} \Delta 50$ ), no disruption by GAGA was discernible (Fig. $4 d$ ). Therefore promoter sequences that include at least two GA/CT elements are necessary for nucleosome displacement when GAGA is competing directly with the nucleosome assembly reaction, and sequences including three to four elements are required for the disruption of a preassembled nucleosome.

\section{ATP requirement}

The assembly of nucleosomes in vitro requires ATP and an energy-regeneration system ${ }^{8}$. We investigated the energy require-

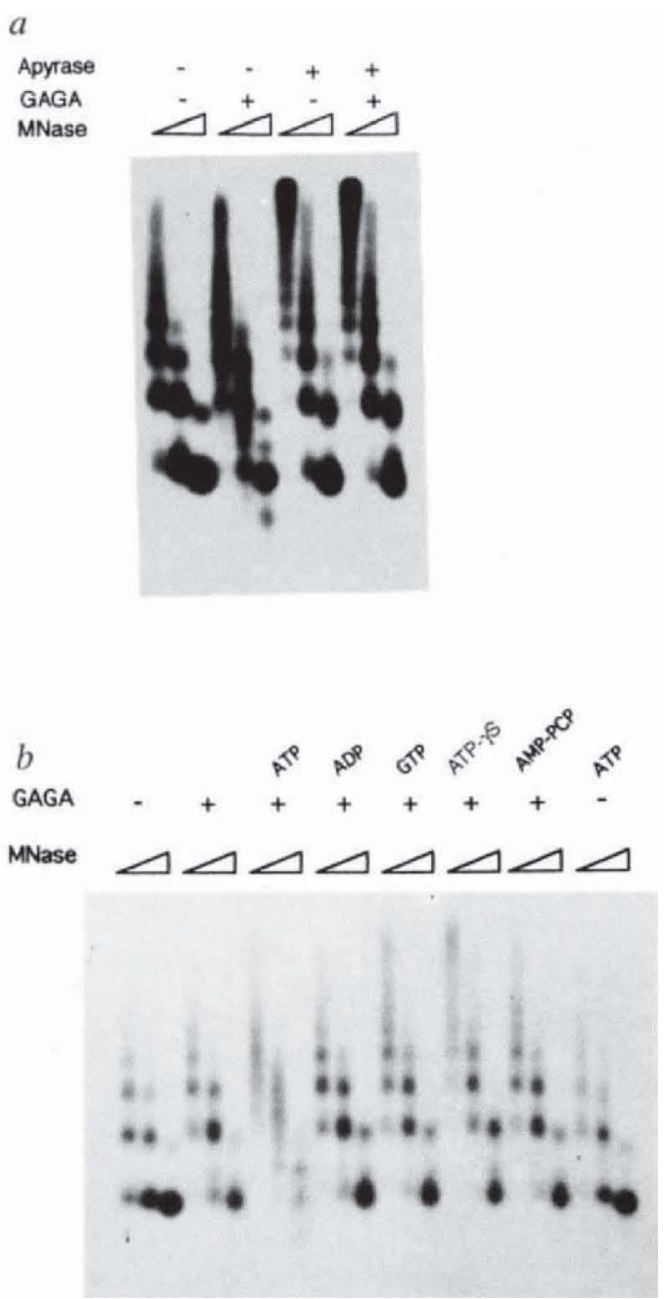

FIG. 5 ATP requirement for GAGA-dependent disruption of chromatin structure. a, Effect of apyrase on chromatin disruption at the hsp70 promoter; hsp70-plasmid chromatin reconstituted with GAGA and treated with apyrase was analysed by MNase digestion. The resultant DNA was blotted and hybridized as for Fig. 1a. $b$, Restoration of nucleosome disruption with supplemental ATP. Partially purified $h s p 70$ chromatin was incubated with GAGA, nucleotides and analogues as indicated, digested with MNase, and processed as described.

METHODS. After $5.5 \mathrm{~h}$ of reconstitution of the $h s p 70$-plasmid in $50 \mu \mathrm{l}$ of chromatin assembly reaction, 0.1 units of apyrase (Sigma, grade VI) was added and incubated at $26{ }^{\circ} \mathrm{C}$ for $15 \mathrm{~min}$ before incubation with GAGA for $30 \mathrm{~min}$, followed by MNase digestion (Fig. 1 legend). For purification of chromatin, 100- $\mu$ aliquots of a 6 -h assembly were applied on 1-ml Bio-Gel A-1.5 m spin columns (prepared in a 1-ml tuberculin syringe) pre-equilibrated with $10 \mathrm{mM}$ HEPES-KOH, $\mathrm{pH} 7.7,50 \mathrm{mM} \mathrm{KCl}$, $0.5 \mathrm{mM}$ EGTA, $5 \mathrm{mM} \mathrm{MgCl}_{2}, 10 \%$ glycerol. $50 \mu \mathrm{l}$ of the fractionated material was incubated at $26^{\circ} \mathrm{C}$ for $30 \mathrm{~min}$ with GAGA and nucleotides $(0.8 \mathrm{mM})$ as indicated
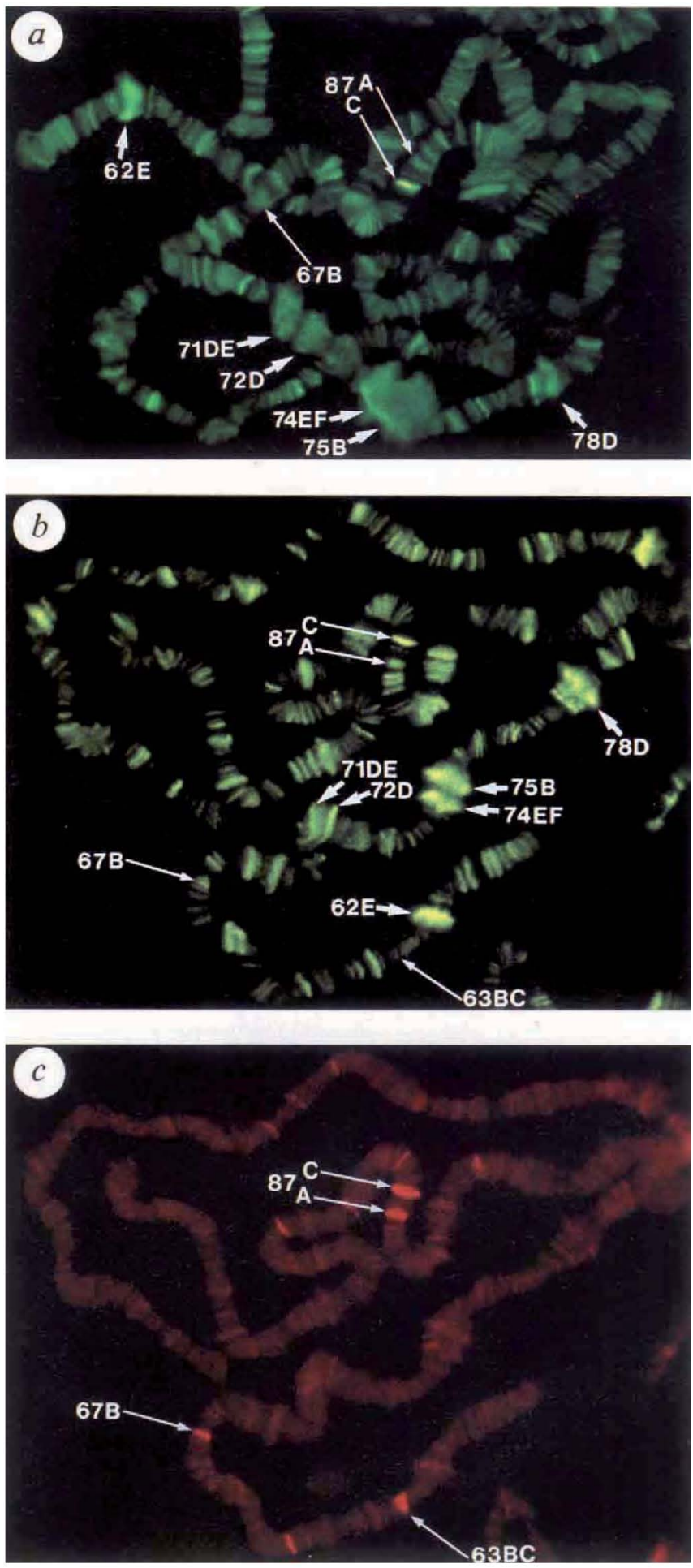

FIG. 6 Localization of GAGA on polytene chromosomes. $a, b$, Indirect immunofluorescent staining for GAGA (fluroescein isothiocyanate, FITC) on polytene chromosomes prepared from $a$, unshocked, and $b$, heatshocked $\left(2 \mathrm{~min}\right.$ at $\left.37^{\circ} \mathrm{C}\right)$ third instar larvae. Loci are indicated that carry $h s p 70$ genes $(87 \mathrm{~A}, 87 \mathrm{C})$, the $h s p 82$ gene $(63 \mathrm{BC})$, the small heatshock genes $h s p 27, h s p 26, h s p 23$ and $h s p 22$ (67B), prominent developmental puffs. $c$, The same preparation as in $b$, stained for HSF with rhodamine.

METHODS. Polytene squashes and chromosome staining were performed as described ${ }^{51}$, using an additional pre-fixing step. Dry milk (Carnation) was substituted for BSA. A 1:500 dilution of rabbit antiserum to GAGA, and a 1:1,000 dilution of mouse antiserum to HSF were used as primary antibodies. 
ments of chromatin disruption by adding the ATP-hydrolysing enzyme apyrase after completion of nucleosome assembly but before introduction of GAGA. Treatment of apyrase substantially suppressed nucleosome disruption by GAGA on the $h s p 70$ promoter (Fig. 5a); treatment of GAGA alone with apyrase did not affect its ability to bind to free DNA in an electrophoretic mobility shift assay (data not shown). Depletion of ATP using hexokinase and glucose also suppressed nucleosome disruption (data not shown). GAGA-dependent nucleosome disruption was restored upon addition of fresh ATP to a reconstituted chromatin template depleted of nucleotides by gel filtration (Fig. $5 b$ ). The ATP in the reaction could not be substituted with GTP, ADP, or with the non-hydrolysable analogues ATP- $\gamma \mathrm{S}$ and AMP-PCP. We conclude that the specific disruption of nucleosome structure by GAGA is facilitated by ATP hydrolysis.

\section{Chromosomal distribution of GAGA}

In addition to the $h s p 70$ and hsp 26 genes, GAGA binds in vitro to a range of housekeeping and developmental genes in Drosophila $^{10}$. We determined the distribution for GAGA in situ by indirect immunofluorescent staining of polytene chromosomes using a polyclonal antiserum specific for GAGA. The distribution of GAGA was essentially identical between chromosome preparations from unshocked or briefly heat-shocked larvae (Fig. $6 a, b$ ). Staining for GAGA was strong at many chromosomal loci, including the $87 \mathrm{C}$ locus, which carries several hsp 70 genes. Staining was moderate at $87 \mathrm{~A}$, which carries two $h s p 70$ genes, and at $67 \mathrm{~B}$, the site of the small heat-shock genes $h s p 27, h s p 26, h s p 23$ and $h s p 22$. Not surprisingly, the staining for GAGA at locus 63BC, which encodes Hsp82 and whose promoter region is deficient in $\mathrm{GA} / \mathrm{CT}$ repeats ${ }^{22}$, is low to undetectable; other trans-acting factors must therefore be responsible for the three DNase I hypersensitive sites and an array of nucleosomes positioned on this heat-shock gene ${ }^{4}$. The co-localization of GAGA and HSF at $87 \mathrm{~A} / 87 \mathrm{C}$ and $67 \mathrm{~B}$ but not at $63 \mathrm{BC}$, was confirmed by staining the chromosome preparation for both factors (Fig. $6 b, c)$. Staining for GAGA was very strong at prominent developmental puffs active in the late larval stage: $62 \mathrm{E}$, 71DE, 72D, 74EF, 75B and $78 \mathrm{D}^{23}$. Among these loci is the $E 74$ gene at $74 \mathrm{EF}$, encoding an ETS-related DNA-binding protein that carries multiple sites for GAGA binding in vitro ${ }^{24}$.

\section{Discussion}

We have shown that the introduction of recombinant GAGA protein during or after the assembly of long nucleosome arrays in vitro leads to a local disruption of chromatin structure at the hsp70 promoter. The binding of GAGA to four sites on this promoter results in nucleosome disruption, the acquisition of DNase I hypersensitivity at the TATA box and the heat-shock control elements, and a rearrangement of adjacent nucleosomes. Considered together with the chromosomal localization of GAGA in vivo at several heat-shock loci under non-stress and heat-stress conditions, our results indicate that this constitutive transcription factor plays a key role in forming an hsp 70 promoter structure that is accessible to the basal transcription factors and activated HSF trimers. The widespread chromosomal distribution of GAGA may reflect a similar functional requirement for other genes in Drosophila. Our findings are consistent with results showing that Drosophila transformants with mutations in upstream GA/CT elements have decreased nuclease hypersensitivity and promoter activity ${ }^{25}$, and with the functioning of GAGA in an in vitro transcription assay ${ }^{26}$ by antirepression rather than direct activation.

How does GAGA mediate its function in chromatin? The disruption of nucleosome structure resulting from GAGA binding can occur in competition with the assembly of nucleosomes as well as on pre-existing nucleosomes. In this respect. disruption can be classified as 'dynamic', in contrast to the 'preemptive' mechanisms ascribed previously to factor-dependent chromatin perturbation in vitro'. Dynamic mechanisms of nucleosome disruption could include dissociation of the nucleosome octamer into the $(\mathrm{H} 3 / \mathrm{H} 4)_{2}$ tetramer and $\mathrm{H} 2 \mathrm{~A} / \mathrm{H} 2 \mathrm{~B}$ dimers, sliding of intact octamers away from promoter sequences by weakening of histone DNA interactions, or removal of histone octamers from the promoter. Protein-protein interactions between GAGA proteins bound at several adjacent sites may also subject the intervening DNA to torsional constraints and contribute to nucleosome destabilization. The 519-residue GAGA open reading frame ${ }^{10}$ shows a single zinc-finger in the Cterminal region, stretches of glutamine residues and basic amino acids, and an $\mathrm{N}$-terminal 120-residue domain with significant sequence homology to Drosophila trans-acting factors tramtrack ${ }^{27-29}$ and the Broad Complex ${ }^{30}$, and kelch ${ }^{31}$, a component of intercellular bridges in Drosophila. It will be interesting to define the structural domains of GAGA responsible for the effects on chromatin.

The dependence on ATP of GAGA-mediated nucleosome disruption suggests a number of energy-dependent mechanisms to alter histone-DNA contacts. GAGA might itself bind ATP and disrupt nucleosome structure by a conformational change; but it contains no canonical ATP-binding motif, neither have we been able to demonstrate ATP-binding activity for this protein. GAGA may act in conjuction with other components in the crude embryo extract that need to hydrolyse ATP for nucleosome disruption. The evolutionarily conserved proteins of the SWI 2 /SNF 2 family are non-DNA-binding mediators that facilitate transcriptional activation by antagonizing the inhibitory effects of chromatin proteins ${ }^{32,33}$. As these proteins share conserved motifs with poxvirus DNA-dependent ATPases ${ }^{34}$, homologous Drosophila proteins such as brahma $a^{35}$ might assist GAGA by fulfilling the ATP-dependent function. Alternatively, other ATP-dependent factors or enzymes affecting nucleosome assembly or spacing (for review, see ref. 36) may act constitutively or be locally concentrated by GAGA to modify or destabilize the nucleosome core particle such that binding of the transcription factor at multiple sites would suffice per se to complete disruption. In this respect, we have noted minor but reproducible effects on nucleosome structure mediated by GAGA binding in extracts depleted of ATP (Fig. 5, and unpublished observations). Before individual components and their relative contributions to nucleosome disruption can be defined and different models distinguished, fractionated material and a defined chromatin substrate will be needed.

Although nucleosome disruption by dynamic competition in vivo is feasible for several yeast trans-acting factors (PHO 4 (ref. 37), GRF-2/REF-1 (refs 38, 39), RAP-1 (ref. 40) and GAL4 (ref. 41)), the means by which disruption can occur has only been investigated in vitro for GAL4, for which nucleosome displacement required the presence of excess carrier DNA and was independent of the GAL4 transactivation domain ${ }^{42}$. In addition, a GAL4-VP16 hybrid protein can relieve nucleosome-mediated repression of transcription in vitro ${ }^{4345}$. The binding of the glucocorticoid receptor in responsive cells has also been implicated in the active process of nucleosome disruption, although this receptor forms a stable ternary complex with nucleosomal DNA in vitro $^{46-48}$. Our finding that GAGA-mediated disruption of existing nucleosomes is facilitated by ATP hydrolysis opens avenues of investigation into how the repressive effects of nucleosome structure at a eukaryotic promoter might be overcome.

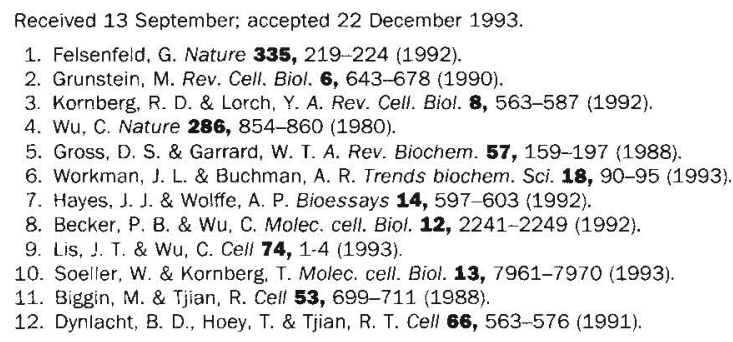


13. Rougvie, A. E. \& Lis, J. T. Cell 54, 795-804 (1988).

15. Gilmour. D. S. Thom, J. Troc. natn. Acad. Sci. U.S.A. 90, $7923-7927$ (1993)

16. Wu, C. Nature 317, 84-87 (1985).

18. Parker C.S. \& Topol, Dell 36, 357-309

19. van Holde, K. E. Chromatin 16-30 (Springer, New York, 1989)

. Elgin, S. C. R. \& Hood, L. E. J. biol. Chem. 12, 4984-4991 (1973).

pringer, Berlin, 1972

25. Lue Q. Wallrath, L L, Granok H. \& Elgin S. C. R. Molec cell 26. Kerrigan, L. A., Croston, G. E., Lira, L. \& Kadonaga, J. T. J. biol. Chem. 266, 574-582

(1991).

27. Harrison, S. D. \& Travers, A. A. EMBO J. 9, $207-216$ (1990)

28. Read, D. Q Maniey, J. L. EMBO J. 11, 1035-1044 (1992).

30. DiBello, P. R. et al. Genetics 129, 385-397 (1991).

1. Xue, F. \& Cooley, L. Cell 72, 681-693 (1993).

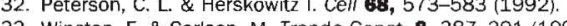

34. Henikoff, S. Trends biochem. Sci 18, 291-292 (1993

36. Aimouzni $G$ \& 2 Wolffe $A$. Exp

37. Schmid, A., Fascher, K. D. \& Horz W. Cell 71, 1-20 (1992).

38. Chasman, D. I. et al. Genes Dev. 4, 503-514 (1990).

39. Morrow, B. E., Ju, Q. \& Warner, J. R. Molec. cell. Biol. 13, 1173-1182 (1993).

42. Workman, J. L. \& kingston, R. E. Science Genes Dev. 7, $857-869(1993)$.

44. Kamakaka, R. T., Buger, M. \& Kadonaga, J. T. Genes Dev. 7, 1779-1795 (1993).

45. Lorch, Y., LaPointe, J. W. \& Kornberg, R. D. Genes Dev. 6, 2282-2287 (1992).

47. Pina, B., Bruggemeier, u. \& Beato, M. Cell 60, 719-731 (1990)

48. Archer, T. K. et al. Molec. cell. Biol. 11, 688-698 (1991.

49. Mason, P. J. et al. J. molec. Biol. 156, 21-35 (1982).

50. Croston, G. E., Lira, L. M. \& Kadonaga, J. T. Prot. Expr. Purific 2, 162-169 (1991).

ACKNOWLEDGEMENTS. We thank W. Soeller and T. Kornberg for the GAGA CDNA clone and for access to data before publication; J. Wisniewski and M. Wisniewska for assistance in 